Methods Patients with UC were prospectively recruited from 11 international centres. Participating endoscopists experienced in IBD received training on PICaSSO before starting the study. The rectum and sigmoid were examined using iScan 1,2\&3 (Pentax, Japan) and inflammatory activity was assessed using Mayo, UCEIS and PICaSSO. Biopsies were taken for histological assessment using Robarts Histological Index (RHI), Nancy, ECAP, Geboes and Villanacci. Follow up data was obtained at 12 months.

Results A total of 307 patients were recruited.The interobserver agreement for the PICaSSO score was 0.879 (95\% CI 0.826-0.924). The PICaSSO total and PICaSSO mucosal scores strongly correlated with histology scores and was statistically better than MES and UCEIS as show in figure 1. When using a PICaSSO total score of $\leq 3$ the AUROC to predict $\mathrm{MH}$ by $\mathrm{RHI}(\leq 3+$ absence of neutrophils $)$ was 0.90 (95\% CI 0.86-0.94) and when we compare the AUROC of Picasso vs Mayo $\mathrm{p}$ was $=0.06$. When using the Nancy score $(\leq 1)$ the AUROC was 0.816 (95\% CI $0.77-0.87)$. A KaplanMeier curve shows a significant favourable survival probability without relapse with a PICASSO score of $\leq 3$ Likelihood ratio test $=26.41, \mathrm{p}<0.0000$.

Conclusions This real-life validation study shows the electronic chromoendoscopy score, PICaSSO, can predict accurately histological healing and long-term remission and can be a useful tool in the management of UC.

\section{BOUGIECAP DILATATION DEVICE: NOVEL ENDOSCOPIC METHOD FOR TREATMENT OF OESOPHAGEAL STRICTURES-RESULTS FROM A MULTICENTRE STUDY}

${ }^{1}$ Patricia Duarte*, ${ }^{2}$ Walter Benjamin, ${ }^{1}$ Praful Patel, ${ }^{1}$ Philip Boger, ${ }^{2}$ Simone Schmidbaur, ${ }^{3}$ David Albers, ${ }^{3}$ Brigitte Schumacher, ${ }^{2}$ Alexander Meining, Imdadur Rahman. 'Southampton Interventional Endoscopy Unit, University Hospital Southampton, UK; ${ }^{2}$ UIm University Hospital, Ulm, Germany; ${ }^{3}$ Elisabethkrankenhaus Hospital, Essen, Germany

\subsection{6/gutjnl-2020-bsgcampus.5}

Introduction A novel dilatation device, BougieCap (Ovesco, Germany), allows both tactile and optic feedback of the dilatation procedure without the need for fluoroscopy. The aim of this study was to assess the safety and efficacy of this device in a prospective cohort of patients.

Methods Patients with benign oesophageal strictures and symptoms of dysphagia were recruited from 3 centres in the UK and Germany for planned dilatation with the BougieCap. The device is a single-use transparent conical cap which is fixed to the tip of the endoscope. Once in place, the endoscope is inserted and positioned in front of the stricture and by pushing forward and rotating with the endoscope, enables the conical cap to dilate the mucosa. The primary outcome measure was the technical success of dilatation. Secondary outcome measures were improvement in symptoms of dysphagia, assessed by the Dysphagia Handicap Index (DHI) before and 14 days after the procedure, and adverse events.

Results 104 patients with benign oesophageal strictures underwent BougieCap dilatation between February 2018 to September 2019. Aetiology of strictures were peptic 63\%, radiation $15 \%$, anastomotic $7 \%$, caustic $6 \%$, EoE 5\%, post-ESD/EMR $4 \%$. Mean diameter of strictures was $5 \mathrm{~mm}( \pm 2.3)$. Bougienage was successful in $97 \%$. In 3 cases, with a long narrow stricture, bougienage failed because of high resistance at the site of the stricture causing buckling of the endoscope in the pharynx. Symptoms of dysphagia improved after bougienage
(53 points Day 0 v 21 points day 14, p< 0.01). No severe adverse events were reported.

Conclusions Endoscopic treatment of benign strictures using the BougieCap is highly successful and safe. It enables direct visual and tactile control of the bougienage procedure with control of mucosal damage within the strictured area. This might help to adapt treatment more precisely to the stricture. Symptoms of dysphagia are improved in short-term follow-up.

\section{O6 ARTIFICIAL INTELLIGENCE USING CONVOLUTIONAL NEURAL NETWORKS FOR DETECTION OF EARLY BARRETT'S NEOPLASIA}

${ }^{1}$ Mohamed Abdelrahim*, ${ }^{2}$ Masahiro Saiko, ${ }^{3}$ Yukiko Masaike, ${ }^{4}$ Shota Ohtsuka, ${ }^{1}$ Ejaz Hossain, 'Sophie Arndtz, ${ }^{1}$ Pradeep Bhandari. 'Queen Alexandra Hospital Portsmouth, Portsmouth, UK; ${ }^{2}$ Biometrics Research Laboratories, NEC, Kanagawa, Japan; ${ }^{3}$ NEC Europe Ltd, London, UK; ${ }^{4}$ NEC Corporation, Tokyo, Japan

\subsection{6/gutjnl-2020-bsgcampus.6}

Initial results from THE PAIGE PROJECT Portsmouth's Project on Artificial Intelligence in Gastrointestinal Endoscopy Introduction Endoscopic detection of early Barrett's neoplasia remains very challenging, with significant inter-observer variation in identifying and assessing these lesions. Artificial intelligence is proposed to help with computer aided detection in this field and could have significant clinical and cost implications. We aim to develop and validate a deep learning (DL) algorithm using Convolutional Neural Networks (CNN) for detection of Barrett's neoplasia.

Methods We collected 132 high definition white light endoscopy images from 46 lesions of histologically confirmed Barrett's neoplasia. These images were marked and annotated using specially designed software, and reviewed by two experts on advanced assessment and management of Barrett's neoplasia. Another 119 images of non dysplastic Barrett's were collected from 20 patients and used as control. Both dysplastic and non dysplastic images were divided into three datasets and used for training, validation and testing of CNN algorithm. We used SegNet segmentation architecture. Graphic processing unit used was 'GeForce RTX 2080 Ti. We collected metrics on processing speed,

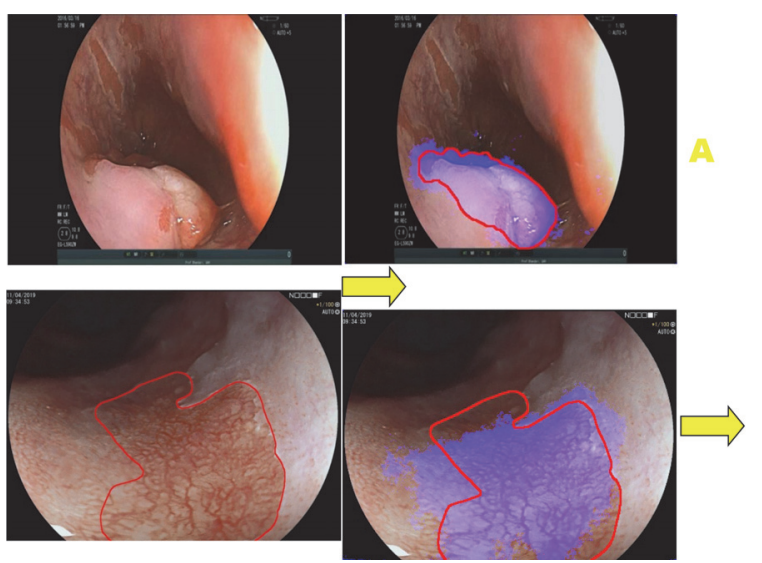

Abstract 06 Figure 1 Two examples of the algorithm prediction in (A) a raised adenocarcinoma lesion, and (B) a flat subtle HGD lesion. The red line is expert marking of the lesion (ground truth), while the blue coloured patch is the algorithm delineation of the lesion. 